\title{
A COMPLEMENT THEOREM IN THE UNIVERSAL MENGER COMPACTUM
}

\author{
R. B. SHER
}

(Communicated by James E. West)

\begin{abstract}
A. Chigogidze has shown that two $Z$-sets in the universal Menger compactum of dimension $k+1$ have the same $k$-shape if and only if their complements are homeomorphic. We show that this result holds for weak $Z$ sets. The class of weak $Z$-sets, defined herein and analogous to the weak $Z$-sets in $Q$, contains but is larger than the class of $Z$-sets. We give some examples of weak $Z$-sets in the universal Menger compactum and in $Q$ that are not $Z$-sets.
\end{abstract}

\section{INTRODUCTION}

In this paper we generalize a result of Chigogidze [2] by establishing the following result about closed subsets of the universal Menger compactum $\mu^{k+1}$.

Theorem. Suppose $X$ and $Y$ are weak $Z$-sets in $\mu^{k+1}$. Then $k-\operatorname{Sh}(X)=$ $k-\operatorname{Sh}(Y)$ if and only if $\mu^{k+1}-X$ and $\mu^{k+1}-Y$ are topologically equivalent.

This is a shape-theoretic complement theorem. For information on related theorems, see [8,9]. The difference between it and Chigogidze's result is that Chigogidze's deals with $Z$-sets rather than weak $Z$-sets. Now the $Z$-sets are quite 'tame', as is shown by the $Z$-set unknotting theorem [1, Corollary 3.1.5]. Thus there is essentially only one $Z$-embedding of a given compactum in $\mu^{k+1}$; on the other hand, the weak $Z$-sets may exhibit a certain degree of 'wildness'. The difference between the two conditions is that of 'local' vs. 'global'. Since shape theory is intended to deal with global rather than local properties of compacta, a condition such as that satisfied by a weak $Z$-set would seem to be appropriate for a shape-theoretic complement theorem. We remark that a similar result holds for the Hilbert cube [8, Theorem 4.1].

For information on Menger manifolds and the theory of $k$-shape, see [1] and [2] respectively. Our notations and conventions will be standard and should cause the reader no difficulty. We only remark that we need not worry about the distinction between the notion of $\mu$-homotopy [1, Definition 2.1.7] and $k$ -

Received by the editors June 8, 1992.

1991 Mathematics Subject Classification. Primary 54C56, 55P55.

Key words and phrases. Universal Menger compactum, complement theorem, $Z$-set, weak $Z$ set, shape theory, $n$-shape theory. 
homotopy [2, Definition 2.1] since all of the mappings considered herein will satisfy the conditions of [2, Proposition 2.3].

\section{NEIGHBORHOODS OF COMPACTA IN $\mu^{k}$-MANIFOLDS}

In this section we show that if $X$ is a closed subset of the compact $\mu^{k}$ manifold $M$, then $X$ has a basis of particularly nice closed $\mu^{k}$-manifold neighborhoods. We begin by recalling a result of Dranishnikov [6] (cf. [1]) on the representation of compact $\mu^{k}$-manifolds as intersections of piecewise linear manifolds.

Let $P$ be a combinatorial manifold whose underlying polyhedron $|P|$ is a compact piecewise linear manifold of dimension $n \geq 2 k+1$. (In [6] attention is restricted to the case $n=2 k+1$, but it is evident that the results hold for larger dimensions as well.) Let $P_{1}=P$. Having defined $P_{i}$, let $P_{i+1}=\operatorname{St}\left(P_{i}^{(k)}, \beta^{2} P_{i}\right)$, where $P_{i}^{(k)}$ denotes the $k$-skeleton of $P_{i}$ and $\beta^{2}$ denotes second barycentric subdivision. Then if $i=1,2, \ldots,\left|P_{i}\right|=N_{i}$ is a compact $n$-manifold with boundary and $\bigcap_{i=1}^{\infty} N_{i}$ is $\mu^{k}$-manifold. Furthermore, every compact $\mu^{k}$-manifold is topologically equivalent to such an intersection, where we may take $n$ as large as we wish. In the following proposition we assume that the $\mu^{k}$-manifold $M$ is $\bigcap_{i=1}^{\infty} N_{i}$ as above.

Lemma 1. For $i=1,2, \ldots, M \cap \partial N_{i}$ is a Z $Z$-set in $M$.

Proof. Let $f: I^{k} \rightarrow M$ be a map and $\varepsilon>0$. We must show that there is a map $g: I^{k} \rightarrow M$ such that $d(f, g)<\varepsilon$ and $g\left(I^{k}\right) \cap \partial N_{i}=\varnothing$. We note that if $j>i$, then $M \cap \partial N_{i} \subset \partial N_{j}$. Fix $j>i$. Now consider the collar neighborhood $U=\left|\operatorname{St}\left(\partial P_{j}, \beta^{2} P_{j}\right)\right|$ of $\partial N_{j}$. Note that the pair $\left(U, U \cap N_{j+1}\right)$ may be identified with $\left(\partial N_{j} \times I,\left(N_{j+1} \cap \partial N_{j}\right) \times I\right)$ where $\partial N_{j}$ is identified with $\partial N_{j} \times\{0\}$. Hence, there is a retraction $r:\left(U, U \cap N_{j+1}\right) \rightarrow\left(\partial N_{j} \times\{1\}\right.$, $\left.\left(N_{j+1} \cap \partial N_{j}\right) \times\{1\}\right)$. Define $\tilde{f}: I^{k} \rightarrow N_{j+1}$ by $\tilde{f}(x)=f(x)$ if $f(x) \notin U$ and $\tilde{f}(x)=r(f(x))$ if $x \in U$. Applying, if necessary, a small general position adjustment to the portion of $I^{k}$ that maps into $U$, we may suppose that $\tilde{f}\left(I^{k}\right)$ fails to intersect $\operatorname{Lk}\left(P_{j+1}^{(k)}, \beta^{1} P_{j+1}\right)$. We note that if $x \in I^{k}$, then $\tilde{f}(x)$ lies in a simplex of $P_{j+1}$ that does not intersect $\partial N_{j}$. We are thus prepared to begin the inductive use of [6, Lemma 3] to 'absorb' $\tilde{f}$ and convert it to a map $g: I^{k} \rightarrow M$ whose image fails to intersect $\partial N_{j}$; cf. the proof of [6, Lemma 4]. If $j$ is sufficiently large, $g$ is an $\varepsilon$-approximation of $f$ and the proof is complete.

Proposition 1. Let $X$ be a closed subset of the compact $\mu^{k}$-manifold $M$. Then $X$ has arbitrarily small closed neighborhoods $W$ such that $W$ is a $\mu^{k}$-manifold and $\operatorname{Fr} W$ is a Z-set in $W$. We may further suppose that $\operatorname{Fr} W$ is also a $\mu^{k}$-manifold.

Proof. Using the notation established above, fix a positive integer $j$. Let $K_{0}$ be the subcomplex of $P_{j}$ consisting of those simplexes of $P_{j}$ that have dimension at most $k$ and are faces of simplexes of $P_{j}$ that intersect $X$. Then $K_{0}$ is a subcomplex of $P_{j}^{(k)}$ and $N=\left|\operatorname{St}\left(K_{0}, \beta^{2} P_{j}\right)\right|$ is a submanifold of $N_{j+1}$ whose interior relative to $N_{j+1}$ contains $X$. It follows from our representation of $M$ as $\bigcap_{i=1}^{\infty} N_{i}$ that $W=M \cap N$ is a $\mu^{k}$-manifold neighborhood of $X$ that is close 
to $X$ if $j$ is chosen sufficiently large. Since the frontier of $W$ lies in $\partial N$, it follows from Lemma 1 that the frontier of $W$ is a $Z$-set in $W$. Since we may assume $n \geq 2 k+2$, we may further assume that $\operatorname{Fr} W$ is a $\mu^{k}$-manifold.

As a result of Proposition 1, a compact subset of a $\mu^{k}$-manifold has a basis of compact $\mu^{k}$-manifolds neighborhoods with $\mu^{k}$-manifold frontiers that are $Z$-sets relative to the neighborhoods. For convenience in what follows, a neighborhood of a compactum in a $\mu^{k}$-manifold will be such a neighborhood.

\section{WeAK $Z$-SETS IN $\mu^{k+1}$-MANIFOLDS}

In this section we characterize our generalization of the notion of $Z$-set. We give three equivalent conditions. One of these (condition (2) below) is homotopy theoretical and is a global condition that is closest in spirit to the usual definition of $Z$-set. Another (condition (1) below) is geometric. It provides a sort of 'regular neighborhood theory' and allows us to prove our complement theorem using techniques that are similar to those used to prove complement theorems in Euclidean spaces. The third condition (condition (3)) is a sort of 'global general position' result. It is easily seen that a $Z$-set must satisfy these conditions.

Suppose $X$ is a compactum lying in the $\mu^{k+1}$-manifold $M, U$ is a closed neighborhood of $X$, and $K$ is a compact $Z$-set lying in the interior of $U$. Then $K$ is a spine of $U$ relative to $X$ if for each neighborhood $V$ of $K$ there exists a homeomorphism $h: M \rightarrow M$ fixed on $K \cup \mathrm{Cl}(M-U)$ such that $h(X) \subset V$ and $h \mid W \simeq^{k} \operatorname{id}_{W}$ in $W$ for each neighborhood $W$ of $X$ such that $U \subset W$. If each neighborhood $U$ of $X$ has a spine relative to $X$, then we say that $X$ has $Z$-spine neighborhoods. It is clear that this condition holds if each member $U$ of a basis of neighborhoods of $X$ has a spine relative to $X$.

Theorem 1. Let $X$ be a closed subset of the $\mu^{k+1}$-manifold $M$. Then the following are equivalent.

(1) $X$ has Z-spine neighborhoods.

(2) For each neighborhood $U$ of $X$, the inclusion $U-X \hookrightarrow U$ is a $k$ homotopy equivalence.

(3) For each neighborhood $U$ of $X$ and $Z$-set $A$ in $M$, there is a homeomorphism $h: U \rightarrow U$ such that $h(x)=x$ for all $x \in \operatorname{Fr} U$ and $h(X) \cap A=\varnothing$.

Proof. We begin with the proof that (1) implies (2). Let $U$ be a neighborhood of $X$, and let $K$ be a $Z$-set in the interior of $U$ that is a spine of $U$ relative to $X$. Let $f: U \rightarrow U$ be a map such that $f \simeq^{k} \operatorname{id}_{U}$ and $f(U) \cap(K)=\varnothing$. Choose a neighborhood $V$ of $K$ such that $V \cap f(U)=\varnothing$, and let $h: U \rightarrow U$ be a homeomorphism such that $h$ is fixed on $K, h(X) \subset V$, and $h \simeq^{k} \mathrm{id}_{U}$. Let $g: U \rightarrow U-X$ be defined by $g(x)=h^{-1}(f(x))$ for all $x \in U$. If $i$ denotes the inclusion of $U-X$ into $U$, then $i g=h^{-1} f \simeq^{k} \operatorname{id}_{U}$. To see that $g i \simeq^{k} \operatorname{id}_{U-X}$, let $P$ be a polyhedron of dimension $\leq k$ and let $\alpha$ be a map of $P$ into $U-X$. Then there is a map $F: P \times I \rightarrow U$ such that $F(x, 0)=\alpha(x)$ and $F(x, 1)=g(\alpha(x))$ for all $x \in P$. Let $W \subset U-F(P \times\{0,1\})$ be a neighborhood of $X$ whose interior contains a $Z$-set $L$ that is a spine of $W$ relative to $X$. Let $r: U \rightarrow U$ be a map fixed on $U-W$ so that $r(U) \cap L=\varnothing$. Choose a neighborhood $S$ of $L$ such that $S \cap r(U)=\varnothing$, and let $h_{0}: U \rightarrow U$ be a homeomorphism such that $h_{0}$ is fixed on $L \cup(U-W)$ and $h_{0}(X) \subset S$. 
Define $F_{0}: P \times I \rightarrow U-X$ by $F_{0}(x, t)=h_{0}^{-1}(r(F(x, t)))$. Then $F_{0}$ is a homotopy joining $\operatorname{id}_{U-X} \alpha$ and $g i \alpha$, so $g i \simeq{ }^{k} \mathrm{id}_{U-X}$.

We now turn to the proof that (2) implies (3). Let $U$ be a neighborhood of $X$ and $A$ be a $Z$-set in $M$. Let $f: U \rightarrow U-X$ be a $k$-homotopy inverse of the inclusion of $U-X$ into $U$. Denote $A \cap U$ by $A_{U}$. Then $f \mid \operatorname{Fr} U \cup A_{U}$ maps $\operatorname{Fr} U \cup A_{U}$ into $U-X$ and is $k$-homotopic to id $\mid \operatorname{Fr} U \cup A_{U}$ in $U$. Since $f \mid \operatorname{Fr} U: \operatorname{Fr} U \rightarrow U-X$ extends to $f \mid \operatorname{Fr} U \cup A_{U}$ and $f \mid \operatorname{Fr} U \simeq^{k}$ id $\mid \operatorname{Fr} U$ in $U-X$, it follows from the $k$-homotopy extension theorem [1, Proposition 2.1.8(iv)] that id $\mid$ Fr $U$ extends to $g: \operatorname{Fr} U \cup A_{U} \rightarrow U-X$ so that $f \mid \operatorname{Fr} U \cup$ $A_{U} \simeq^{k} g$ in $U-X$. Since $\operatorname{Fr} U$ is a $Z$-set in $U$, we may assume by the $Z$-set approximation theorem [1, Theorem 2.3.8] that $g$ is a $Z$-embedding. Since Fr $U \cup A_{U}$ is a $Z$-set in $U$ and $g \simeq^{k}$ id $\mid \operatorname{Fr} U \cup A_{U}$, we may apply the $Z$ unknotting theorem [1, Theorem 3.1.4] to obtain a homeomorphism $h^{-1}: U \rightarrow$ $U$, such that $h^{-1}(x)=x$ for all $x \in \operatorname{Fr} U$, and

$$
h^{-1}\left(A_{U}\right)=g\left(A_{U}\right) \subset M-X \text {. }
$$

Then $h$ is the desired homeomorphism demonstrating that (2) implies (3).

We now complete the proof by showing that (3) implies (1). We may suppose $M$ is compact and shall once again use the notation established in $\S 2$, where we take $n$ to be at least $2 k+4$. Arbitrarily close to $X$ we may find, as in the proof of Proposition 1, a submanifold $N$ of $N_{j+1}$ so that $M \cap N$ is a neighborhood of $X$ in $M$. Recall that $N$ has a spine $K=\left|K_{0}\right|$, where $K_{0}$ is a subcomplex of $P_{j}^{(k+1)}$. By [6, Lemma 5] $K$ is a $Z$-set in $M$. Letting $U=M \cap N$, we complete the proof by showing that $U$ has a $Z$-spine relative to $X$.

Let Fr $N$ denote the frontier of $N$ relative to $N_{j}$. To begin, we use (3) and Lemma 1 to move $X$ off of $M \cap \operatorname{Fr} N$ by a homeomorphism $f$ of $U$ onto itself that fixes the frontier of $U$ relative to $M$. We shall show that $K$ is a spine of $U$ relative to $f(X)$. It is then easily seen that $f^{-1}(K)$ is a spine of $U$ relative to $X$. For ease of notation, we may simply assume then that $X$ fails to intersect $\operatorname{Fr} N$. Let $V$ be a neighborhood of $K$. Now, by iterating the process of twice barycentrically subdividing and taking derived neighborhoods, we find a regular neighborhood $Q_{0}$ of $K$ in $N$ so that $M \cap Q_{0} \subset V$. We also obtain in this way a regular neighborhood $Q_{1}$ of $\operatorname{Fr} N$ so close to $\operatorname{Fr} N$ that $X \cap Q_{1}=\varnothing$. By regular neighborhood theory (see, e.g., [7]) $Q_{1}$ and $\operatorname{Cl}\left(N-Q_{0}\right)$ are each topologically equivalent to $(\operatorname{Fr} N) \times I$ and there is a homeomorphism of $N$ onto itself that is isotopic to the identity, is fixed on $K \cup F r N$, and carries $Q_{1}$ onto $\mathrm{Cl}\left(N-Q_{0}\right)$. By $[1, \S 2.8]$, the restriction of this homeomorphism to $Q_{1}$ can be used as a 'guide map' to obtain a homeomorphism $h_{1}$ of $M \cap Q_{1}$ onto $M \cap \mathrm{Cl}\left(N-Q_{0}\right)$ that fixes points of the frontier of $M \cap Q_{1}$ in $M$ and that is $k$-homotopic to the inclusion of $M \cap Q_{1}$ into $M \cap \mathrm{Cl}\left(N-Q_{0}\right)$. By Lemma 1 and $Z$-set unknotting, we may suppose that $h_{1}$ carries $M \cap Q_{1} \cap \mathrm{Cl}\left(N-Q_{1}\right)$ onto $M \cap \operatorname{Fr} Q_{0}$. By the $k$-homotopy extension theorem, $h_{1}$ extends to a map $H: N \cap M \rightarrow N \cap M$ such that $H \simeq^{k} \mathrm{id}_{N \cap M}$. Using the fact that $Q_{0}$ is a retract of $N$, we may 'retract and absorb' and thereby suppose that $H \mid M \cap \mathrm{Cl}\left(N-Q_{1}\right)$ carries $M \cap \mathrm{Cl}\left(N-Q_{1}\right)$ into $Q_{0}$. By another adjustment we convert $H$ to a homeomorphism which we then extend to $M$ via the identity to obtain a homeomorphism $h: M \rightarrow M$ so that $h$ is fixed on $K \cup \mathrm{Cl}(M-U), h(X) \subset V$, and $h \mid W \simeq^{k} \operatorname{id}_{W}$ in $W$ for each neighborhood $W$ of $X$ such that $U \subset W$. This completes the proof. 
A closed subset $X$ of the $\mu^{k+1}$-manifold $M$ will be said to be a weak $Z$-set if $X$ satisfies the conditions of Theorem 1 .

The machinery is now in place to prove the main theorem. The notion of 'spine' has been carefully chosen so that the proof is nearly the same as in the $Q$-manifold case, only using the appropriate results for $\mu^{k+1}$ rather than $Q$. We refer the reader to $[8, \S 4]$ for details.

\section{Some EXAMPLES OF WeAK $Z$-SETS IN $Q$ AND $\mu^{k+1}$}

In this section we construct some examples of weak $Z$-sets in $Q$ and $\mu^{k+1}$ that are not $Z$-sets. While it is possible to treat these two ambient spaces separately, it will be convenient to use a result of Chigogidze that allows us to carry out the constructions in $Q$ (where they are somewhat easier to describe) and then transfer to $\mu^{k+1}$ by a certain map. We thank A. Chigogidze for this suggestion. First we recall Chigogidze's result [3, Corollary 1.4].

Theorem C. There exists a $(k+1)$-invertible $U V^{k}$-surjection $\alpha: \mu^{k+1} \rightarrow Q$ such that

(1) there is a subspace $A$ of $\mu^{k+1}$ such that $\alpha \mid A: A \rightarrow Q$ is $(k+1)$-soft and $\mu^{k+1}-A$ is a $\sigma Z$-set in $\mu^{k+1}$, and

(2) if $Z$ is a $Z_{k+1}$-set in $Q$, then $\alpha^{-1}(Z)$ is a $Z$-set in $\mu^{k+1}$.

Recall that a map $\alpha: X \rightarrow Y$ is $n$-invertible if whenever $f: Z \rightarrow Y$ is a map of an at-most $n$-dimensional space $Z$ into $Y$, then $f$ lifts to a map $g: Z \rightarrow X$. If we further require that for each closed subspace $Z_{0}$ of $Z$ and map $h: Z_{0} \rightarrow X$ such that $\alpha h=f \mid Z_{0}$ that $h$ extends to a lift of $f$, then we say that $\alpha$ is $n$-soft. Also, a compactum $Z$ in $Q$ is a $Z_{n}$-set if maps into $Q$ of $I^{n}$ (and hence of compacta of dimension at most $n$ ) can be approximated by maps whose images miss $Z$. Throughout the remainder of the paper, we shall let $\alpha$ be a fixed map as in Theorem C.

Lemma 2. (i) If $W$ is a compactum in $Q$ and $W$ is not a $Z_{k+1}$-set in $Q$, then $\alpha^{-1}(W)$ is not a $Z$-set in $\mu^{k+1}$.

(ii) If $W$ is a weak $Z$-set in $Q$, then $\alpha^{-1}(W)$ is a weak $Z$-set in $\mu^{k+1}$.

Proof. To prove (i), suppose $W$ is a compactum in $Q$ and $\alpha^{-1}(W)$ is a $Z$-set in $\mu^{k+1}$. Let $f: I^{k+1} \rightarrow Q$ be a map. Then $f$ lifts to $g: I^{k+1} \rightarrow \mu^{k+1}$. Since $\alpha^{-1}(W)$ is a $Z$-set, $g$ can be approximated by a map whose image misses $\alpha^{-1}(W)$. Thus, by continuity of $\alpha, f$ can be approximated by a map whose image misses $W$. This shows that $W$ is a $Z_{k+1}$-set in $Q$.

For (ii), suppose $W$ is a weak $Z$-set in $Q$ and let $U$ be a neighborhood of $\alpha^{-1}(W)$ in $\mu^{k+1}$. We shall show that the inclusion $i: U-\alpha^{-1}(W) \hookrightarrow U$ is a $k$-homotopy equivalence. It suffices to assume that $U$ is open and saturated with respect to $\alpha$. Let $V=\alpha(U)$. Since $W$ is a weak $Z$-set in $Q$, there is a homotopy $H: V \times I \rightarrow V$ such that $H(V \times\{1\}) \cap W=\varnothing, H(x, t)=x$ if $t=0$, and $H$ has compact support. Define $f: U \rightarrow Q$ by $f(x)=H(\alpha(x), 1)$. Then, since $\alpha$ is $(k+1)$-invertible, there exists a map $g: U \rightarrow \mu^{k+1}$ such that $\alpha g=f$. Considering $g$ to be a map from $U$ into $U-\alpha^{-1}(W)$, we claim that $g$ is a $k$-homotopy inverse of $i$. 
First we show that $i g \simeq^{k} \mathrm{id}_{U}$. So, let $P$ be a polyhedron of dimension $\leq k$ and let $p: P \rightarrow U$ be a map. We need to show that $p \simeq p g$ in $U$. Recall that $\mu^{k+1}-A$ is a $\sigma Z$-set in $\mu^{k+1}$. It follows that we may, by a slight adjustment if necessary, suppose that $p(K) \subset A$. Now, the homotopy $H$ leads us to a homotopy $F: P \times I \rightarrow V$ such that $F(x, 0)=\alpha p(x)$ and $F(x, 1)=$ $\alpha g p(x)$ for all $x$ in $P$. Since $\alpha$ is $(k+1)$-soft on $A$, there exists a homotopy $G: P \times I \rightarrow U$ such that $G(x, 0)=p(x)$ and $G(x, 1)=g p(x)$ for all $x$ in $P$.

We must now show that $g i \simeq^{k} \mathrm{id}_{U-\alpha^{-1}(W)}$. So, let $P$ be a polyhedron of dimension $\leq k$ and let $p: P \rightarrow U-\alpha^{-1}(W)$ be a map. We need to show that $p \simeq g i p$ in $\bar{U}-\alpha^{-1}(W)$. As above, we may suppose that $p(K) \subset A$. We use the homotopy $H$ and the fact that $W$ is a weak $Z$-set in $Q$ to obtain a homotopy $F: P \times I \rightarrow V-W$ such that $F(x, 0)=\alpha p(x)$ and $F(x, 1)=\alpha g p(x)$ for all $x$ in $P$. The proof is completed by lifting $F$ as in the preceding paragraph.

Example 1. We describe an uncountable family of mutually inequivalently imbedded Hilbert cubes in $Q$ each of which is a weak $Z$-set but not a $Z_{0^{-}}$ set. Let $p=(x, 0) \in I^{2}$, where $0<x<1$. Let $\left\{a_{i}\right\}$ and $\left\{b_{i}\right\}$ be strictly increasing positive real sequences converging to $x$ such that $a_{j}<b_{j}<a_{j+1}$ for all $j \geq 1$, and let $\left\{t_{i}\right\}$ be a strictly decreasing positive real sequence converging to 0 with $t_{1}<1$. Let $C_{i}=\left[a_{i}, b_{i}\right] \times\left[0, t_{i}\right]$. We say that the collection $\left\{C_{i} \mid i \in Z^{+}\right\}$is a sequence of boxes converging to $p$ from the left. We may similarly define, in the obvious way, a sequence of boxes converging to $p$ from the right. Now fix a strictly increasing positive real sequence $\left\{x_{i}\right\}$ converging to 1 . Let $p_{i}=\left(x_{i}, 0\right)$. Let $U_{i}$ be an open neighborhood of $p_{i}$ so that if $i \neq j$, then $U_{i} \cap U_{j}=\varnothing$ and so that the sequence of diameters of the $U_{i}$ 's converges to 0 . Let $\Lambda$ be the set of all sequences of 0 's and 1's, and let $s=\left\{m_{i}\right\} \in \Lambda$. If $m_{i}=0$, construct a sequence of boxes converging to $p_{i}$ from the left and lying in $U_{i}$; if $m_{i}=1$, construct a sequence of boxes converging to $p_{i}$ from the right and lying in $U_{i}$. Then the union of $[0,1] \times\{0\}$ with all of the boxes so constructed is a compact absolute retract that we shall denote by $X_{s}$. Let $Z_{s}=X_{s} \times Q \subset I^{2} \times Q$, and identify $I^{2} \times Q$ with $Q$. Then $Z_{s} \cong Q$. It is easy to see, since $X_{s}$ is the intersection of nice disks in $I^{2}$ that meet the boundary of $I^{2}$ regularly, that $Z_{s}$ is a weak $Z$-set in $Q$. Since $Z_{s}$ has nonempty interior, $Z_{s}$ is not a $Z_{0}$-set in $Q$. It is also easy to see that if $t \in \Lambda$ and $t \neq s$, then $Z_{s}$ and $Z_{t}$ are not equivalently embedded in $Q$.

Example 2. We could proceed as in Example 1 to obtain an uncountable family of inequivalently embedded Menger $(k+1)$-spaces in $\mu^{k+1}$ each of which is a weak $Z$-set but not a $Z$-set. Alternatively, we may apply Lemma 2 to see that the inverse images under $\alpha$ of the sets constructed above are weak $Z$-sets in $\mu^{k+1}$ that are not $Z$-sets. While these may not be homeomorphic to $\mu^{k+1}$, it can be seen that no two are equivalently embedded in $\mu^{k+1}$ when $k \geq 1$.

Example 3. We construct an arc in $Q$ that is a weak $Z$-set but not a $Z_{2}$-set. Begin by letting $Y$ be an arc in $Q$ whose complement is not simply connected. Let $Q^{\prime}$ be the cone on $Q$, which we consider to be the set $(Q \times I) /(Q \times\{1\})$. Then $Q^{\prime} \cong Q$. Let $Y^{\prime} \subset Q^{\prime}$ be the image of $Y \times\left[\frac{1}{2}, 1\right]$, and let $G$ be the upper semicontinuous decomposition of $Q^{\prime}$ whose nondegenerate elements are the subsets of $Y^{\prime}$ corresponding to the sets $Y \times\{t\}$, where $\frac{1}{2} \leq t<1$. Let 
$Q^{\prime \prime}$ be the decomposition space $Q^{\prime} / G$. Note that $Q^{\prime \prime}$ can be considered to be the space obtained by adjoining $Q^{\prime}$ and an arc $K$ via a map from a disk in $Q^{\prime}$ to $K$. Thus, $Q^{\prime \prime}$ is a compact absolute retract. By [5, Theorem 7.1], we can conclude that $Q^{\prime \prime} \cong Q$ provided $Q^{\prime \prime}$ has the Disjoint Disks Property. This latter property may be verified much as in the proof of [4, Main Lemma]. In $Q^{\prime \prime}$, the image of $Y^{\prime}$ is an arc $Z$ that is cellular (since $Y^{\prime}$ is cellular in $Q^{\prime}$ ), so by [8, Corollary 4.7] $Z$ is a weak $Z$-set. Note that there are loops in $Q^{\prime}-Y^{\prime}$ that are inessential but cannot be shrunk in the complement of $Q^{\prime}-\left(Q \times\left[0, \frac{1}{2}\right]\right)$. This clearly implies that $Z$ is not a $Z_{2}$-set in $Q^{\prime \prime}$.

Example 4. We now construct an arc in $\mu^{k+1}, k \geq 1$, that is a weak $Z$-set but not a $Z$-set. Let $K$ denote the arc in $Q$ constructed in Example 3. Let $G$ denote the upper semicontinuous decomposition of $\mu^{k+1}$ whose members are the sets $\alpha^{-1}(p)$, where $p \in K$, and singleton sets in the complement of $\alpha^{-1}(K)$. By the Sum Theorem for Dimension, the space $\mu^{k+1} / G$ is $k+1$ dimensional. It follows from [1, Theorem 4.3.1] that $\mu^{k+1} / G$ is topologically $\mu^{k+1}$ provided $\mu^{k+1} / G$ can be shown to have the Disjoint $(k+1)$-Cells Property $\left(D D^{k+1} P\right)$. To see that $\mu^{k+1} / G$ satisfies $D D^{k+1} P$, let $f, g: I^{k+1} \rightarrow \mu^{k+1} / G$ be maps. Denote the natural projection of $\mu^{k+1}$ onto $\mu^{k+1} / G$ by $\pi$, and denote $\pi\left(\alpha^{-1}(K)\right)$ by $L$. Approximately lift $f$ and $g$ to maps $f^{\prime}, g^{\prime}: I^{k+1} \rightarrow \mu^{k+1}$ so that $\pi f^{\prime}$ and $\pi g^{\prime}$ are close to $f$ and $g$ respectively. We may assume, by slight adjustment, that $f^{\prime}\left(I^{k+1}\right)$ and $g^{\prime}\left(I^{k+1}\right)$ lie in $A$. We then slightly adjust the maps $\alpha f^{\prime}: I^{k+1} \rightarrow Q$ and $\alpha g^{\prime}: I^{k+1} \rightarrow Q$, limited by a small cover of $K$, to obtain maps $f^{\prime \prime}, g^{\prime \prime}: I^{k+1} \rightarrow Q$ so that $f^{\prime \prime}\left(I^{k+1}\right) \cap g^{\prime \prime}\left(I^{k+1}\right) \cap K=\varnothing$ and so that $f^{\prime \prime}$ and $g^{\prime \prime}$ agree with $\alpha f^{\prime}$ and $\alpha g^{\prime}$, respectively, outside of a small neighborhood of the inverse image of $K$. Using the properties of $\alpha$, we lift $f^{\prime \prime}$ and $g^{\prime \prime}$ to maps $\tilde{f}, \tilde{g}: I^{k+1} \rightarrow \mu^{k+1}$ so that $\pi \tilde{f}$ and $\pi \tilde{g}$ are close to $\pi f^{\prime}$ and $\pi g^{\prime}$ (hence, close to $f$ and $g$ ) and so that $\pi \tilde{f}\left(I^{k+1}\right) \cap \pi \tilde{g}\left(I^{k+1}\right) \cap L=\varnothing$. Using the fact that $\mu^{k+1} / G-L$ is a $\mu^{k+1}$-manifold, we may now make a final adjustment, working in the complement of $L$, to complete the approximation of $f$ and $g$ by maps whose images are disjoint. Thus, $\mu^{k+1} / G \cong \mu^{k+1}$. It can now be seen that $L$ is not a $Z$-set in $\mu^{k+1} / G$, for if $L$ were a $Z$-set, then the techniques used above could be used to show that $K$ is a $Z_{2}$-set in $Q$. It also can be seen, since $\alpha^{-1}(K)$ is a weak $Z$-set in $\mu^{k+1}$, that $L$ is a weak $Z$-set in $\mu^{k+1} / G$.

\section{REFERENCES}

1. M. Bestvina, Characterizing $k$-dimensional universal Menger compacta, Mem. Amer. Math. Soc., No. 380, Amer. Math. Soc., Providence, RI, 1988.

2. A. Chigogidze, Compacta lying in the n-dimensional universal Menger compactum and having homeomorphic complements in it, Mat. USSR-Sb. 61 (1988), 471-484.

3. _ $U V^{n}$-equivalence and n-equivalence, Topology Appl. 45 (1992), 283-291.

4. R. J. Daverman, Embedding phenomena based upon decomposition theory: wild Cantor sets satisfying strong homogeneity properties, Proc. Amer. Math. Soc. 75 (1979), 177-182.

5. R. J. Daverman and J. J. Walsh, Čech homology characterizations of infinite dimensional manifolds, Amer. J. Math. 103 (1981), 411-435.

6. A. Dranishnikov, On resolutions of $L C^{n}$-compacta, Geometric Topology and Shape Theory (S. Mardešić and J. Segal, eds.), Lecture Notes in Math., vol. 1283, Springer-Verlag, New York, 1987, pp. 48-59. 
7. J. F. P. Hudson, Piecewise linear topology, W. A. Benjamin, New York, 1969.

8. R. B. Sher, Complement theorems in shape theory, Shape Theory and Geometric Topology (S. Mardešić and J. Segal, eds.), Lecture Notes in Math., vol. 870, Springer-Verlag, New York, 1981, pp. 150-168.

9. R. B. Sher, Complement theorems in shape theory, II, Geometric Topology and Shape Theory (S. Mardešić and J. Segal, eds.), Lecture Notes in Math., vol. 1283, Springer-Verlag, New York, 1987, pp. 212-220.

Department of Mathematics, University of North Carolina at Greensboro, GreensBoro, NoRTh CAROLINA 27412

E-mail address: sherrb@iris.uncg.edu 